\title{
Comportamiento de la vigilancia epidemiológica de la leptospirosis humana en Colombia, 2007-2011
}

\author{
Solmara Bello', Milena Rodríguez², Andrea Paredes², Fredy Mendivelso³, Diana Walteros², \\ Flor Rodríguez ${ }^{1}$, María Elena Realpe ${ }^{1}$ \\ ${ }^{1}$ Grupo de Microbiología, Subdirección Red Nacional de Laboratorios, Instituto Nacional de Salud, Bogotá, D.C., \\ Colombia \\ 2 Grupo de Zoonosis, Subdirección de Vigilancia y Control en Salud Pública, Instituto Nacional de Salud, Bogotá, \\ D.C., Colombia \\ ${ }^{3}$ Grupo de Enfermedades no Transmisibles, Subdirección de Vigilancia y Control en Salud Pública, Instituto \\ Nacional de Salud, Bogotá, D.C., Colombia
}

Introducción. La leptospirosis es una zoonosis reemergente de distribución mundial causada por una espiroqueta del género Leptospira. Durante los últimos años en Colombia aumentó el número de casos en humanos y animales.

Objetivo. Caracterizar epidemiológicamente los casos de leptospirosis notificados al Sistema Nacional de Vigilancia en Salud Pública de Colombia y hacer una aproximación para conocer los serogrupos que circulan en el país.

Materiales y métodos. Se diseñó un estudio observacional de corte retrospectivo, con registros del proceso de vigilancia de los casos reportados por el software Sivigila y muestras enviadas al Grupo de Microbiología de la Red Nacional de Laboratorios, durante el periodo 2007 a 2011. Se registraron variables de tipo sociodemográficas y se analizaron 17 serogrupos de Leptospira. En el análisis se utilizaron medidas de frecuencia, tendencia central y dispersión.

Resultados. Se procesaron 11.786 registros, confirmándose 4.621 casos de leptospirosis. Las entidades territoriales con mayor registro fueron Valle del Cauca, Antioquia, Risaralda, Atlántico y Barranquilla; y las de incidencia más alta fueron Guaviare, Risaralda, San Andrés, Santa Marta y Barranquilla. El mayor número de casos reportados perteneció al área urbana, con mayor frecuencia de hombres (77\%), estudiantes (19,4\%) y amas de casas (13,6 \%), con una mediana por edad de 29 años (rango intercuartílico: 45-19). Se evidenció la circulación de 17 serogrupos en el país; los más frecuentes fueron Australis (24,89\%), Hebdomadis (9,33\%) y Sejroe $(8,0 \%)$.

Conclusión. En Colombia se ha mejorado la notificación y clasificación final de los casos, lo que ha permitido identificar al serogrupo Australis como el de mayor circulación.

Palabras clave: leptospirosis, Colombia, microaglutinación (MAT), vigilancia en salud pública. doi: http://dx.doi.org/10.7705/biomedica.v33i0.1608

Epidemiological surveillance of human leptospirosis in Colombia, 2007-2011

Introduction: Leptospirosis is a reemerging zoonosis of worldwide distribution, caused by a spirochete of the genus Leptospira. In Colombia, the disease represents a major public health issue, and there has been an increased number of cases in humans and animals.

Objective: To characterize epidemiologically cases of leptospirosis reported to the National Public Health Surveillance in Colombia, and to make an approach to determine the serogroups circulating in the country.

Materials and methods: A retrospective observational study was designed using a process of monitoring records, which included cases reported by the software SIVIGILA and samples sent to the Microbiology Group of the National Laboratory Network (GM-RNL), for the period 2007-2011. We registered socio-demographic variables and analyzed 17 serogroups of Leptospira.

Results: A total of 11,786 records were processed, with 4,621 confirmed cases of leptospirosis. The geographic places which reported the highest number of cases were: Valle del Cauca, Antioquia,

\footnotetext{
Contribución de los autores:

María Elena Realpe y Diana Walteros colaboraron en los procesos de seguimiento del reporte de casos y emisión de resultados de laboratorio.

Flor Rodríguez y Andrea Paredes contribuyeron en el procesamiento de las muestras y la escritura del artículo.

Fredy Mendivelso participó en la depuración de la base de datos, análisis, escritura del artículo y correcciones.

Solmara Bello y Milena Rodríguez fueron las investigadoras principales del proyecto, realizaron la depuración de la base de datos, eñ análisis de los resultados, la edición y la corrección del artículo.
} 
Risaralda, Atlántico and Barranquilla, and those with the highest incidence were Guaviare, Risaralda, San Andres, Santa Marta and Barranquilla. The largest number of cases was from urban areas, and more commonly in men (77\%), students (19.4\%) and housewives (13.6\%). A median age of 29 years (IQR 45-19) was observed. There was evidence of 17 serogroups circulating in the country, from which the three most frequent were Australis (24.89\%), Hebdomadis (9.33\%) and Sejroe (8.0\%).

Conclusions: In Colombia, the reported cases have improved as well as their final classification, allowing us to determine the Australis serogroup as the most widely circulating one.

Keywords: leptospirosis, Colombia, MAT, public health surveillance.

doi: http://dx.doi.org/10.7705/biomedica.v33i0.1608

La leptospirosis se considera una zoonosis emergente y reemergente de distribución mundial, causada por una bacteria perteneciente al género Leptospira, la cual se adquiere al tener contacto directo o indirecto con orina infectada de animales, suelos, aguas o alimentos contaminados (1-4).

El reservorio universal es la rata (Rattus); asimismo, existen otros reservorios como cánidos, suidos, bóvidos y murciélagos. Se presenta con más frecuencia en hombres que en mujeres y se dice que la razón es de 9:1 (3-5).

En países asiáticos, como Filipinas, y otros de Centroamérica y Suramérica, como Honduras, Cuba, Brasil y Colombia, los casos en humanos son adquiridos en los hogares en zonas urbanas 0 por actividades de trabajo en zonas rurales (6-9). Actualmente, se reconoce la enfermedad como un problema creciente de salud pública a nivel mundial, la cual crea una mayor preocupación en los países de Latinoamérica, debido al aumento en el número de casos tanto en humanos como en animales (10-12).

Según lo referido por Eduardo Vedor de Paula y otros autores, las actividades ocupacionales, recreativas y el fenómeno del cambio climático, están relacionados con la presentación y el aumento de casos a nivel mundial (Vedor de Paula E. Anais XII Simposio Brasilero de Sensoramiento Remoto. Goiania, Brasil: INPE; 16-21 de abril de 2005. p. 2301-8) $(6,13)$.

La Organización Mundial de la Salud (OMS) reporta una incidencia anual de leptospirosis estimada en 0,1 casos por 100.000 habitantes para climas templados, de 10 a 100 por 100.000 habitantes en climas tropicales y 100 por 100.000 habitantes

\section{Correspondencia:}

Solmara Bello, Grupo Microbiología, Subdirección Red Nacional de Laboratorios, Instituto Nacional de Salud, Avenida Calle 26 $N^{\circ}$ 51-20, Bogotá, D.C., Colombia

Teléfono: (571) 2207700 , extensión 1421o@ins.gov.co

Recibido: 09/05/12; aceptado:03/04/13 en brotes y grupos de alto riesgo. Los países como Brasil, los del sudeste Asiático y China, tienen la mayor notificación de casos humanos de leptospirosis (14).

Existe una clasificación tradicional en la que se especifica que todas las especies de Leptospira biflexa son saprofitas, mientras que Leptospira interrogans incluye todas las especies patógenas de leptospira. En la actualidad, mediante el análisis de hibridación de ADN, se han clasificado las leptospiras patógenas en nueve especies patógenas y seis especies intermedias, y seis especies de leptospiras saprofitas (7). Todas las especies reconocidas de Leptospira se clasifican en 24 serogrupos que están contemplados en 260 serovariedades o serotipos; esta clasificación se basa en los lipopolisacáridos presentes en la superficie celular (15). La International Leptospirosis Society (ILS) recomienda el uso de una batería de 19 serotipos, que cubren 17 serogrupos para su diagnóstico, mediante la técnica de microaglutinación (MAT). Esta técnica permite determinar los anticuerpos aglutinantes de clase $\operatorname{lgM}$ e $\lg G$ en el suero de un paciente (16).

En Colombia, la leptospirosis hace parte de las zoonosis de notificación obligatoria al Sistema Nacional de Vigilancia en Salud Pública desde el año 2007. Es una enfermedad con un menor número de casos reportados si se compara con otros síndromes febriles, como dengue, malaria y hepatitis. Sin embargo, ha cobrado mayor interés para las autoridades sanitarias, especialmente por el incremento de casos registrados durante las temporadas de lluvia e inundaciones presentadas en Colombia (10,11,17-21).

Se han identificado debilidades en el proceso de vigilancia, como son la dificultad en el acceso a las pruebas de diagnóstico y el desconocimiento de la enfermedad en todas sus áreas (manejo, cuadro clínico, diagnóstico, tratamiento), lo que resulta en un subregistro de casos y sesgos en la información (8). 
Teniendo en cuenta lo anterior, el objetivo de este estudio fue caracterizar epidemiológicamente los casos de la enfermedad que fueron notificados al Sistema Nacional de Vigilancia en Salud Pública de Colombia y, así, identificar los serogrupos de mayor circulación en el país.

\section{Materiales y métodos}

\section{Diseño del estudio y población objeto}

Se diseñó un estudio observacional retrospectivo sobre los registros recolectados durante el proceso de vigilancia, mediante las fichas de notificación y los reportes entregados por el Laboratorio Nacional de Referencia para el periodo de 2007 a 2011.

Se utilizaron las definiciones de caso establecidas en el protocolo nacional de vigilancia en salud pública de Colombia. Un caso probable se define como el paciente que presenta fiebre, cefalea y mialgias, asociado a uno o más síntomas o manifestaciones que sugieran progresión de la enfermedad, acompañado de antecedentes epidemiológicos sugestivos, como exposición a inundaciones o contacto con aguas estancadas por actividad laboral o recreativa y contacto con roedores o animales enfermos. Se consideran actividades de riesgo laboral la recolección de basuras, la limpieza de arroyos, y el trabajo en aguas, ganadería y agricultura. Un caso se considera confirmado por laboratorio si la muestra procesada por la técnica de microaglutinación presenta títulos de 1:400 o mayores, o si existe un aumento de dos veces los títulos en caso de disponer de muestras pareadas (8).

Se registraron variables de tipo sociodemográficas como edad, sexo, procedencia, ocupación, tipo de afiliación a salud, entre otras; además, se analizaron los serotipos involucrados y el número de muestras procesadas, de los casos confirmados por laboratorio. La identificación de Los serogrupos y serotipos se identificaron mediante la técnica de microaglutinación, en las muestras recibidas por el Laboratorio de Microbiología de la Red Nacional de Laboratorios del Instituto Nacional de Salud. Inicialmente, se efectuó la prueba con seis y, posteriormente, con 28 serotipos (Australis, Bratislava, Autumnalis, Rachmati, Ballum, Castellonis, Bataviae, Canicola, Celledoni, Cynopteri, Grippotyphosa, Hebdomadis, Copenhageni, Icterohaemorrhagiae, Javanica, Poi, Panamá, Pomona, proechimys, Pyrogenes, Gorgas, Guaricura, Hardjo, Saxkoebing, Sejroe, Wolffi, Shermani y Tarassovi); estos que pertenecen a 17 serogrupos (Australis, Autumnalis,
Ballum, Bataviae, Canicola, Celledoni, Cynopteri, Grippotyphosa, Hebdomadis, Icterohaemorrhagiae, Javanica, Panamá, Pomona, Pyrogenes, Sejroe, Shermani y Tarassovi). Las cepas fueron donadas por el centro de referencia Royal Tropical Institute (KIT Amsterdam The Netherlands). Para verificar la pureza de las cepas, se practicó la prueba de absorción cruzada y se participó en un control de calidad anual (The International Proficiency Testing Scheme for the Leptospirosis MAT), con el National Reference Laboratory for Leptospira (NRL) del Royal Tropical Institute, obteniéndose resultados satisfactorios.

\section{Análisis estadístico}

Para el análisis de datos se tomaron los casos confirmados por vigilancia y laboratorio; se usaron medidas de frecuencia en variables cualitativas y, medidas de tendencia central y dispersión, para datos cuantitativos. Los datos fueron recolectados en una base de Excel $\AA$, y se usaron el software SPSS18® (PASW Statistics) para análisis estadístico y el SIGEpi® en el proceso de análisis de la información geográfica.

\section{Resultados}

Se recolectaron por el sistema de vigilancia, en total, 11.786 registros $(2.858,24,2 \%$ por laboratorio, 8.135, $69 \%$, por reportes al sistema de vigilancia y $793,6,8 \%$, por ambas fuentes) de casos procedentes de las 36 entidades territoriales

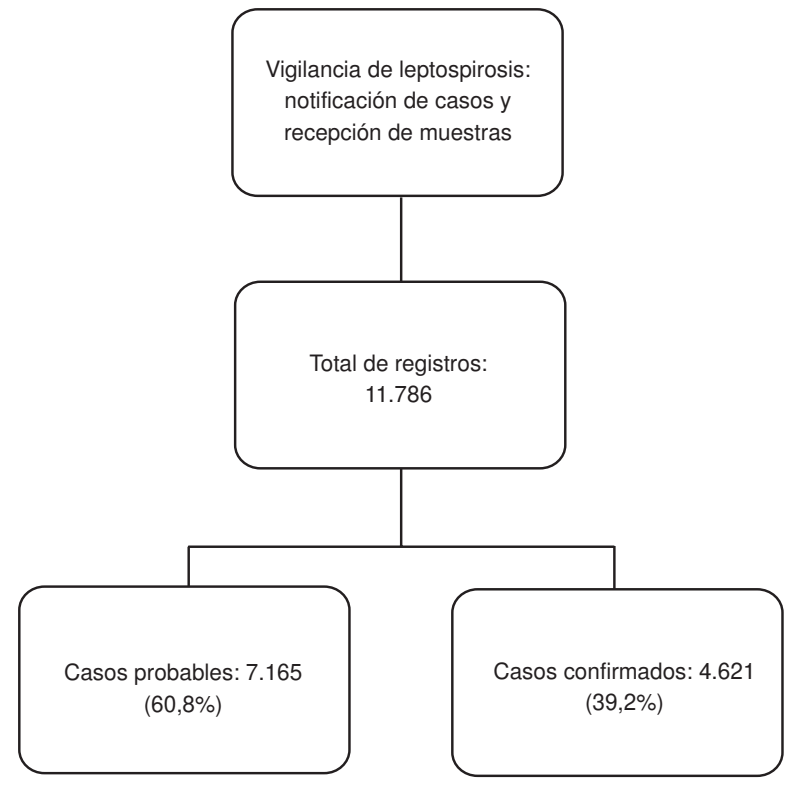

Figura 1. Flujograma de información de casos de leptospirosis, Colombia 2007-2011 
de Colombia (32 departamentos y 4 distritos: Santa Marta, Barranquilla, Cartagena y Bogotá) (figura 1).

Durante el año 2010 se presentó el mayor número de reportes al sistema de vigilancia con 3.295 casos (28\%) y, según el envío de muestras al Grupo de Microbiología, se encontró que durante el 2011 se recibió el mayor número de muestras pareadas $(169,35,21 \%)$. Del total de casos registrados, 48,6 $\%$ (7.165) fueron probables y 31,3\% (4.621) fueron confirmados. Del total de confirmados, se recibieron 378 (8\%) muestras en el laboratorio de referencia; de estas, el 39,4\% (149) fueron pareadas y el 60,6 $\%$ (229) fueron únicas (figuras 2 y 3 ).

La tasa de incidencia acumulada para el periodo de estudio fue de 10,27 casos por 100.000 habitantes y la incidencia más alta se observó en el año 2010, con 2,9 casos por 100.000 habitantes (figura 4). Las regiones que presentan mayor número de casos son la Andina-Pacífica, con 2.673 casos (57 \%) y los cinco departamentos/distritos que se destacan por su alta incidencia son: Guaviare con 267,3, Risaralda con 46,4, San Andrés con 45,4, Santa Marta con 36,9 y Barranquilla con 27,24 casos por 100.000 habitantes (figura 5).

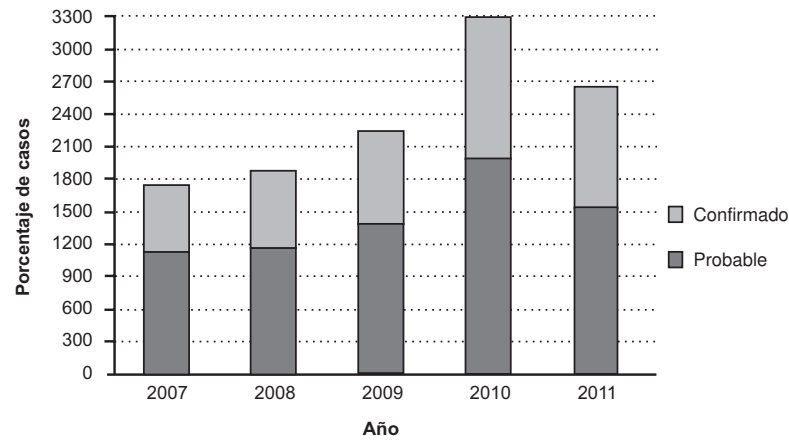

Figura 2. Clasificación por tipo de caso de leptospirosis por año

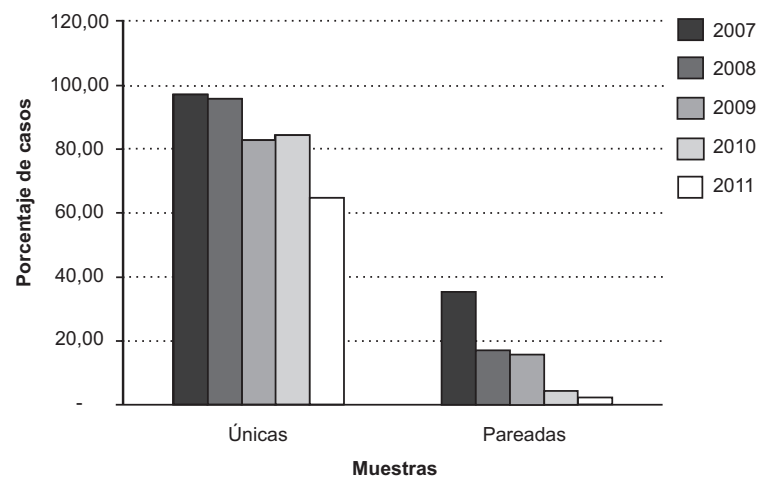

Figura 3. Porcentaje de muestras recibidas por el Grupo Microbiología del Laboratorio Nacional de Referencia, según año de envío

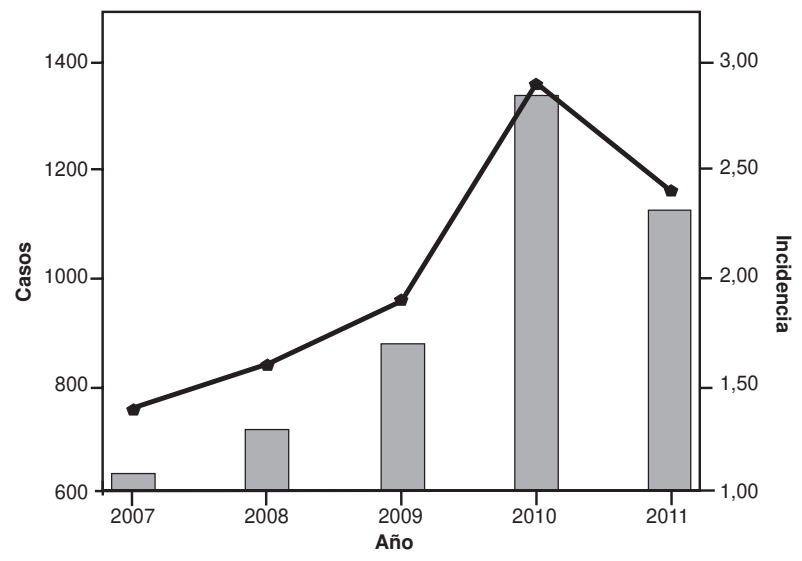

Figura 4. Total de casos de leptospirosis y tasa de incidencia (casos confirmados/100.000 habitantes)

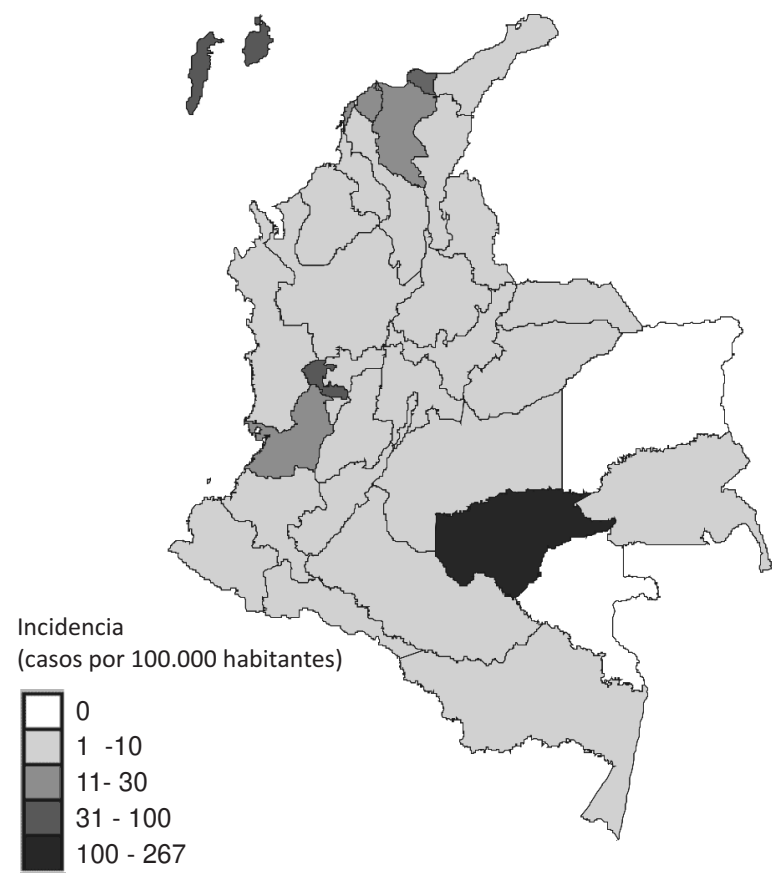

Figura 5. Proporción de incidencia de leptospirosis, Colombia 2007-2011

Respecto a la distribución por sexo, la mayoría de los pacientes con leptospirosis eran hombres, con $69,2 \%$ (3.196), con una razón hombre-mujer de 2:1. La mediana de la edad para los pacientes confirmados fue de 29 años (rango intercuartílico: 45-19); el grupo de edad más afectado se encontró entre los 20 y 24 años, con 593 casos (12,8\%), y la mayor incidencia por grupos quinquenales fue de 14,88 casos por 100.000 habitantes (figura 6 ).

La mayoría de los casos se presentaron en zona urbana (66,5 \%; 3072), en estudiantes (19,4 $\%$; 895) y personas que realizaban actividades relacionadas con el hogar-amas de casa-(13,6\%; 
624). El $40 \%$ (1.860) de los casos se encontraban afiliados al régimen subsidiado y el $23 \%$ eran no afiliados (cuadro 1).

Los serogrupos más frecuentes fueron Australis $(24,89 \%)$, Hebdomadis $(9,33 \%)$ y Sejroe $(8 \%)$, demostrándose así una variabilidad de circulación de serogrupos (cuadro 2).

Cuadro 1. Características sociodemográficas de casos confirmados de leptospirosis, Colombia, 2007-2011

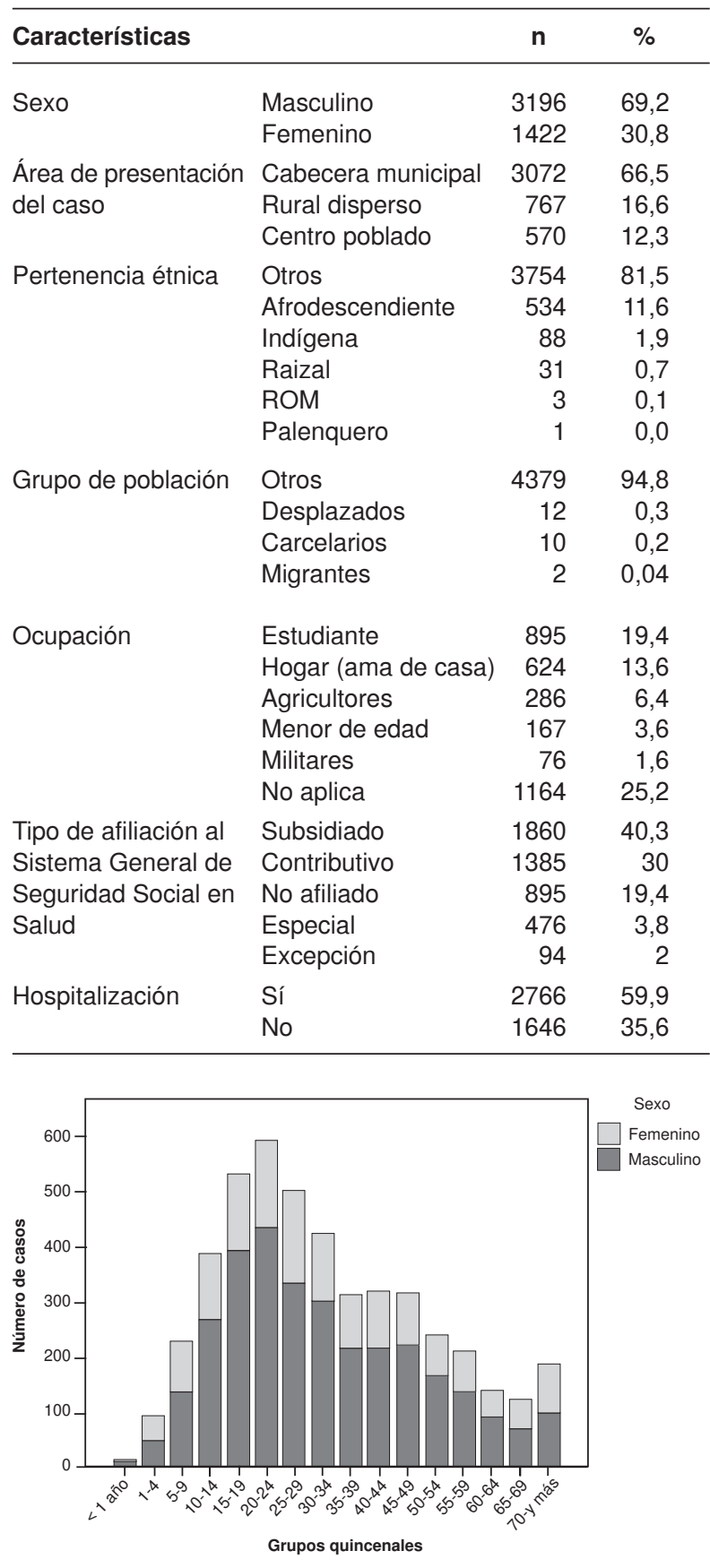

Figura 6. Casos de leptospirosis por grupo de edad y sexo

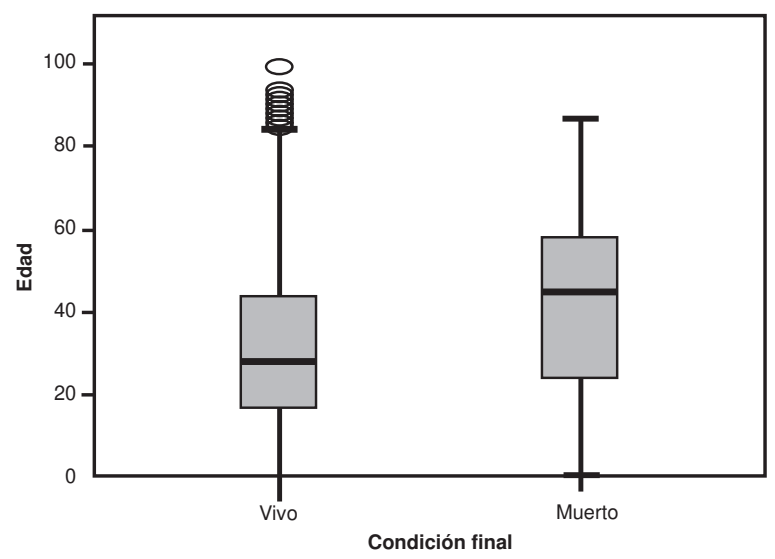

Figura 7. Distribución de casos de leptospirosis, según condición final y edad, Colombia 2007-2011

En cuanto a la mortalidad, se presentaron 209 muertes en el periodo de estudio, que representan el $1,7 \%$ de la notificación total. El $55 \%$ (116) de los casos fueron confirmados por laboratorio, obteniéndose una letalidad de 4,5\%. La mediana de edad en los casos fallecidos es de 47 (rango intercuartílico: 59-22) (figura 7).

\section{Discusión}

A pesar de encontrarse un aumento en la notificación de casos año tras año, este cambio no puede atribuirse a una mayor presentación de casos de la enfermedad, debido a las actividades desarrolladas para el fortalecimiento de la vigilancia epidemiológica, mediante el proceso de sensibilización e implementación del diagnóstico, dirigidas a las secretarías de salud.

El mayor incremento en el reporte de casos se evidenció en el último trimestre del 2010, durante la estación lluviosa; esta relación se ha documentado en la literatura científica como un factor de riesgo para la enfermedad $(9,12,16)$.

En el periodo de estudio el bajo porcentaje de muestras pareadas recibidas inicialmente $(13,7 \%)$, afectó directamente la confirmación de casos. Esta situación mejoró durante los años analizados en el presente estudio, alcanzándose un porcentaje total de $31,3 \%$, acorde con lo reportado por países como Argentina que, para el 2011, según los datos del Ministerio de Salud, confirmaron el $14 \%$ de los casos reportados. En su último informe de leptospirosis 2007, Perú reportó 16 $\%$ de casos confirmados. El país que reportó un mayor porcentaje de confirmación fue Brasil (47\%) (9,12,22-24).

Se observó una mayor presentación de casos en hombres, pero la razón hombre-mujer (2:1) difiere 
Cuadro 2. Circulación de serogrupos de Leptospira por entidad territorial, Colombia, 2007-2011

\begin{tabular}{|c|c|c|c|c|c|c|c|c|c|c|c|c|c|c|c|c|c|c|c|}
\hline Entidad territorial & 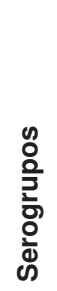 & 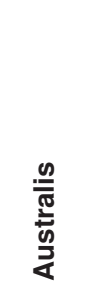 & 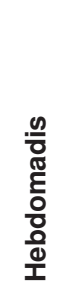 & 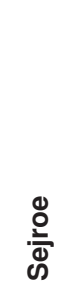 & 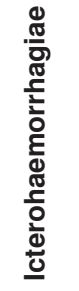 & 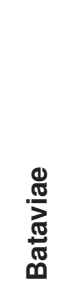 & 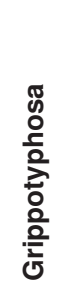 & 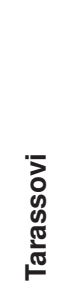 & 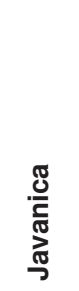 & 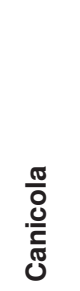 & $\begin{array}{l}\overline{\bar{\varpi}} \\
\text { हूँ } \\
\bar{\Phi} \\
\bar{\sigma}\end{array}$ & 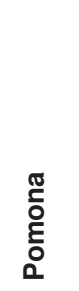 & 巨్ & 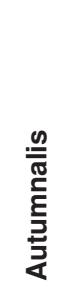 & 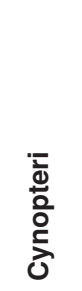 & 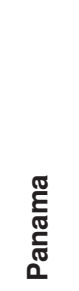 & 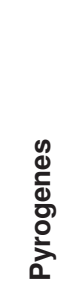 & $\begin{array}{l}\overline{\bar{c}} \\
\overline{0} \\
\overline{\bar{d}} \\
\bar{d}\end{array}$ & 픔 \\
\hline Antioquia & & 7 & 0 & 4 & 6 & 2 & 5 & 2 & 4 & 1 & 2 & 1 & 1 & 3 & 1 & 1 & 2 & 1 & 43 \\
\hline Atlántico & & 1 & & & 4 & & 1 & 1 & & & 1 & & & & & 1 & & & 9 \\
\hline Bogotá & & 1 & 1 & 1 & & 1 & & & & & & & & & & & & & 4 \\
\hline Bolívar & & 1 & & & & & 1 & & & & & 1 & & & & & 1 & 1 & 5 \\
\hline Boyacá & & & & & & 1 & & & & & & 1 & & & & & & & 2 \\
\hline Caldas & & & & & & & & 1 & & & & & & & & & & & 1 \\
\hline Caquetá & & & & & & & & & & & & 1 & & & & & & & 1 \\
\hline Casanare & & 1 & & & & & & & & & & & & & & & & & 1 \\
\hline Cesar & & & & & & & & & 1 & & & & & & & & & & 1 \\
\hline Córdoba & & & 1 & 1 & & & & & & & & & & & & & & & 2 \\
\hline Guainía & & 1 & & & & & & & & & & & & & 1 & & & & 2 \\
\hline Guajira & & & 2 & 1 & & & & & & & & & & & & & & & 3 \\
\hline Guaviare & & 3 & & & 1 & & 1 & & & & & & & & & & & & 5 \\
\hline Huila & & 13 & 4 & 1 & & 1 & 2 & 3 & 1 & & & 3 & 2 & 1 & & & 1 & & 32 \\
\hline Magdalena & & & & & & & & & & 1 & & & & & & 2 & & & 3 \\
\hline Meta & & 1 & & & & & & & & & & & & & & & & & 1 \\
\hline Nariño & & 2 & & & & & 1 & 1 & & & 1 & 1 & & & 1 & & & & 7 \\
\hline Norte de Santander & & & & & 1 & & & & & & & & & & & & & & 1 \\
\hline Putumayo & & 2 & 1 & & & 1 & & 2 & & 1 & 1 & & 1 & & 2 & & & & 11 \\
\hline Risaralda & & 1 & 1 & 2 & 2 & 4 & & 1 & 3 & 5 & 1 & & & 2 & & 1 & & 1 & 24 \\
\hline San Andrés & & & & & & & & & & & & & 3 & & & & & & 3 \\
\hline Santander & & 7 & 1 & 4 & 3 & 2 & 1 & 1 & 1 & 2 & 2 & & & 1 & 1 & 1 & & & 27 \\
\hline Sucre & & 14 & 10 & 4 & & 3 & & & 2 & & & & 1 & & & & 2 & & 36 \\
\hline Tolima & & 1 & & & & & & & & & & & & & & & & & 1 \\
\hline Total & & 56 & 21 & 18 & 17 & 15 & 12 & 12 & 12 & 10 & 8 & 8 & 8 & 7 & 6 & 6 & 6 & 3 & 225 \\
\hline Porcentaje & & 24,89 & 9,33 & 8,00 & 7,56 & 6,67 & 5,33 & 5,33 & 5,33 & 4,44 & 3,56 & 3,56 & 3,56 & 3,11 & 2,67 & 2,67 & 2,67 & 1,33 & 100 \\
\hline
\end{tabular}

de lo reportado en la literatura científica $(9: 1,7: 1)$, lo cual puede relacionarse con la mayor frecuencia de pacientes amas de casa, probablemente por contaminación intradomiciliaria (25). En este estudio se obtiene un alto porcentaje de casos procedentes de la zona urbana, contrario a lo descrito en otros países, en los cuales los pacientes proceden con mayor frecuencia de zonas rurales debido a la ocupación (agricultores, veterinarios, etc.) $(21,26)$.

Se observó una alta incidencia en la distribución de casos en los departamentos de Guaviare y San Andrés, teniéndose en cuenta que la población de estas entidades territoriales corresponde al 0,23\% y $0,16 \%$, respectvamente. Es importante destacar que, aproximadamente desde el año 2004, Guaviare inició una vigilancia centinela enfocada en síndromes febriles, por lo cual su notificación siempre ha sido la mayor en su región, destacándose entre todos los departamentos o distritos del país.
Se reportó circulación de 17 serogrupos en el país, observándose mayor frecuencia del serogrupo Australis, seguido de Hebdomadis. De acuerdo con la literatura científica, el serogrupo Australis se asocia principalmente a suidos, mientras que el serogrupo Hebdomadis se asocia a bóvidos, ratas y roedores $(27,28)$. Estos serogrupos se reportan en otros países de la región de Asia, como Tailandia y Sri Lanka, a diferencia de la India e Indonesia, los cuales no los reportan $(26,29,30)$. En países de Suramérica, como Perú, se observa un comportamiento muy similar; se reporta Australis como uno de los principales y Argentina reporta el serogrupo Sejroe como el principal $(23,26,30)$. Según los resultados obtenidos en este estudio, en Colombia se encontró como el cuarto serogrupo de mayor circulación.

De acuerdo con los resultados obtenidos, concluimos que existe un aumento progresivo en el proceso de 
notificación de casos de la enfermedad en el país, situación similar a la presentada en otros países de la región. Todavía se observan dificultades en la disponibilidad y oportunidad para la toma de muestras pareadas de laboratorio, requisito indispensable para la confirmación de los casos, constituyéndose como el mayor reto de las acciones del sector. De forma similar a lo reportado en diferentes estudios, las personas jóvenes y económicamente activas representan el grupo con mayor frecuencia de la enfermedad. Llama la atención que un importante porcentaje de casos en el país se presentó en áreas de residencia urbana, contrario a lo reportado en la literatura científica mundial, donde predominan los casos provenientes de zonas rurales.

Se demostró circulación de los 17 serogrupos en el país, lo cual, explica las diferencias ecológicas y ambientales que tenemos en los diferentes departamentos. ElserogrupoAustralisestáasociado principalmente con los suidos, lo que indica que el manejo de este reservorio es uno de los mayores riesgos de contagio de la enfermedad.

Se recomienda llevar a cabo en el futuro diversos estudios que permitan identificar la relación huéspedreservorio, para poder establecer programas de intervención adecuados para la prevención de esta enfermedad.

\section{Agradecimientos}

A las secretarías de salud departamentales y distritales de Colombia, a los subdirectores de Vigilancia y Control en Salud Pública y Red Nacional de Laboratorios del Instituto Nacional de Salud, y demás autoridades, quienes facilitaron el proceso de investigación.

\section{Conflicto de intereses}

Los autores manifiestan que no existen conflictos de intereses en la elaboración y ejecución de este proyecto.

\section{Financiación}

El trabajo realizado estuvo financiado por el Instituto Nacional de Salud.

\section{Referencias}

1. Katz A, Ansdell V, Effler P, Middleton C, Sasaki D. Leptospirosis in Hawaii, 1974-1998: Epidemiologic analysis of 353 laboratory-confirmed cases. Am J Trop Med Hyg. 2002;66:61-70.

2. Meites E, Jay MT, Deresinski S, Shieh WJ, Zaki SR, Tompkins L, et al. Reemerging Leptospirosis, California.
Emerg Infect Dis. 2004;10:406-12. http://dx.doi.org/10.3201/ eid1003.030431

3. Romero EC, Bernardo CC, Yasuda PH. Human leptospirosis: A twenty-nine-year serological study in São Paulo, Brazil. Rev Inst Med Trop Sao Paulo. 2003;45:245-8. http://dx.doi.org/10.1590/S0036-46652003000500002

4. Ciceroni L, Stepan E, Pinto A, Pizzocaro P, Dettori G, Franzin L, et al. Epidemiological trend of human leptospirosis in Italy between 1994 and 1996. Eur J Epidemiol. 2000;16:79-86.

5. Michael A, Matthias M, Díaz M, Campos K, Calderón M, Michael R, et al. Diversity of bat-associated Leptospira in the Peruvian Amazon inferred by bayesian phylogenetic analysis of $16 \mathrm{~S}$ ribosomal DNA sequences. Am J Trop Med Hyg. 2005;73:964-74.

6. Oliveira DS, Guimarães MJ, Portugal JL, Medeiros Z. The socio-demographic, environmental and reservoir factors associated with leptospirosis in an urban area of northeastern Brazil. Ann Trop Med Parasitol. 2009;103:149-57. http://dx.doi.org/10.1179/136485909X398221

7. Cerqueira GM, McBride AJ, Queiroz A, Pinto LS, Silva EF, Hartskeerl RA, et al. Monitoring Leptospira strain collections: The need for quality control. Am J Trop Med Hyg. 2010:82:83-7. http://dx.doi.org/10.4269/ajtmh.2010.090558

8. Instituto Nacional de Salud. Subdirección de Vigilancia y Control en Salud Pública. Sivigila. Colombia: Protocolo de Vigilancia y Control de Leptospirosis, 2011. Fecha de consulta: 7 de abril de 2012. Disponible en: http://www.ins. gov.co/lineas-de-accion/Subdireccion-Vigilancia/sivigila/ Protocolos\%20SIVIGILA/LEPTOSPIROSIS.pdf

9. Lau CL, Smythe LD, Craig SB, Weinstein P. Climate change, flooding, urbanization and leptospirosis: Fuelling the fire? Trans R Soc Trop Med Hyg. 2010;104:631-8. http:// dx.doi.org/10.1016/j.trstmh.2010.07.002.

10. Epstein PR, Calix Pena O, Blanco Racedo J. Climate and disease in Colombia. Lancet. 1995;346:1243-4. http:// dx.doi.org/10.1016/S0140-6736(95)91856-6

11. Sebek Z, Sixl W, Valova M, Marth E, Köck M, Reinthaler FF. Serological investigations for leptospirosis in humans in Columbia. Geogr Med Suppl. 1989;3:51-60.

12. Román $\mathbf{H}$, Terán $\mathbf{F}$, Álvarez W. Descripción epidemiológica de un brote de leptospirosis en el departamento de Río San Juan, Nicaragua. Mayo-julio 1999. Fecha de consulta: 14 de julio de 2009. Disponible en: http://desastres.cies. edu.ni/ninformativas/2007/leptospirosis_roman_teran_ alvarez.pdf

13. Katz AR, Buchholz AE, Hinson K, Park SY, Effler PV. Leptospirosis in Hawaii, USA, 1999-2008. Emerg Infect Dis. 2011;17:221-6. http://dx.doi.org/10.3201/eid1702. 101109

14. Leptonet. Royal Tropical Institute, Amsterdam, The Netherlands. Incidencia anual de leptospirosis. Fecha de consulta: 21 de marzo 2012. Disponible en: http://www. leptonet.net/html/incident_map.asp

15. Evangelista KV, Coburn J. Leptospira as an emerging pathogen: A review of its biology, pathogenesis and host immune responses. Future Microbiol. 2010;5:1413-25. http://dx.doi.org/10.2217/fmb.10.102 
16. Organización Mundial de la Salud, Organización Panamericana de la Salud y Sociedad Internacional de Leptospirosis. Leptospirosis humana: guía para el diagnóstico, vigilancia y control. 2008. Fecha de consulta: 10 de abril 10 2012. Disponible en: http://www.med.monash. edu.au/microbiology/staff/adler/guia-esp.pdf.

17. Ochoa J, Sánchez A, Ruiz I. Epidemiología de la leptospirosis en una zona andina de producción pecuaria. Rev Panam Salud Pública. 2000;7:325-31. http://dx.doi.org/10. 1590/S1020-49892000000500006

18. Orrego A, Giraldo G, Ríos B, Valencia P. Leptospirosis en personas de riesgo de quince explotaciones porcinas y de la central de sacrificio de Manizales, Colombia. Arch Med Vet. 2003;2:205-13. http://dx.doi.org/10.4067/S0301732X2003000200008

19. Ferro B, Rodríguez A, Pérez M, Travi B. Seroprevalencia de infección por Leptospira en habitantes de barrios periféricos de Cali. Biomédica. 2006;26:250-7.

20. Agudelo P, Restrepo B, Arboleda M. Leptospirosis en el Urabá antioqueño, Colombia. Cad Saude Pública. 2007;23:2094-104.http://dx.doi.org/10.1590/S0102311X2007000900017

21. Ríos R, Franco S, Mattar S, Urrea M, Tique V. Seroprevalencia de Leptospira sp., Rickettsia sp. Ehrlichia $\mathrm{sp}$. en trabajadores rurales del departamento de Sucre, Colombia. Infectio. 2008;12:318-23.

22. Ministerio de Salud, Presidencia de la Nación de Argentina. Boletín integrado de la vigilancia SE02. Fecha de consulta: 21 de marzo de 2012. Disponible en: http:// www.msal.gov.ar/index.php/home/boletin-integrado-dvigilancia.
23. Instituto Nacional de Salud de Perú. Informe de situación de la leptospirosis en el Perú 2007. Fecha de consulta: 21 de marzo de 2012. Disponible en: http://www.ins.gob.pe/ repositorioaps/0/4/jer/-1/leptospirosis/informe situaciónlept ospirosisp\%C3\%ba2007.pdf

24. Ministério de Saúde do Brasil. Datasus. Leptospirosescasos confirmados notificados no sistema de informasão de agravos de notificação. Fecha de consulta: 21 de marzo de 2012. Disponible en: http://dtr2004.saude.gov.br/sinanwed/ tabnet/dh?sinannet/lepto/bases/leptobrnet.def.

25. Levett PN. Leptospirosis. Clin Microbiol Rev. 2001;14:296326. http://dx.doi.org/10.1128/CMR.14.2.296-326.2001

26. Vanasco NB, Schmeling MF, Lottersberger J, Costa F, Ko Al, Tarabla HD. Clinical characteristics and risk factors of human leptospirosis in Argentina (1999-2005). Acta Trop. 2008;107:255-8.http://dx.doi.org/10.1016/j.actatropica. 2008. 06.007.

27. Céspedes M, Balda L, González D, Tapia R. Situación de La leptospirosis en El Perú 1994-2004. Rev Peru Med Exp Salud Pública. 2006;23:56-65.

28. Hartskeerl RA, Terpstra WJ. Leptospirosis in wild animals. Vet Q. 1996;18(Suppl.3):149-50.

29. World Health Organization. Leptospirosis situation in the WHO South- East Asia Region. Fecha de consulta: 12 marzo de 2012. Disponible en: http://www.searo.who.int/linkfiles/ communicable_disease_surveillance_and_response-seacd-216.pdf.

30. Céspedes M. Leptospirosis: enfermedad zoonótica reemergente. Rev Perú Med Exp Salud Pública. 2005;22:290-307. 